\title{
RDUS
}

Revue de DROIT UNIVERSITÉ DE SHERBROOKE

Titre : $\quad$ L'INCERTITUDE DES PROBABILITÉS EN DROIT ET EN MÉDECINE

Auteur(s): $\quad$ Katherine LIPPEL

Revue: $\quad$ RDUS, 1991-1992, volume 22, numéro 2

Pages: $\quad 445-472$

ISSN : $\quad 0317-9656$

Éditeur: $\quad$ Université de Sherbrooke. Faculté de droit.

URI : $\quad$ http://hdl.handle.net/11143/13424

DOI : $\quad$ https://doi.org/10.17118/11143/13424 
Page vide laissée intentionnellement. 


\section{L'INCERTITUDE DES PROBABILITÉS EN DROIT ET EN MÉDECINE*}

par Katherine LIPPEL**

Le droit, la science et la médecine interagissent souvent à l'occasion du contentieux d'indemnisation, mais les conséquences de cette interaction sont souvent ignorées. Chacune de ces disciplines interprète différemment des notions de base comme la probabilité, la causalité, et la certitude. La confusion qui en découle porte préjudice, d'une manière systémique, aux droits des personnes qui revendiquent une indemnisation. Le fardeau de preuve doit être défini en fonction de la pensée juridique, et ne devrait pas se confondre avec la notion de probabilité scientifique. Les règles de preuve traditionnelles devront être scrutées afin de les adapter aux besoins d'un système d'indemnisation où les enjeux médico-légaux et le déséquilibre entre les parties génèrent un accès inégal aux données scientifiques ou médicales. La «neutralité» et la réserve judiciaire peuvent d'elles-mêmes créer une situation de partialité lorsque l'inégalité des parties est de nature structurelle.

Law, medicine and science are often interacting elements in the adjudication of compensation claims. Yet the consequences of this interaction are often overlooked. Concepts basic to these disciplines, such as probability, causation, and certainty, have very different meanings in the respective fields. Confusion as to the meaning actually used creates an ongoing handicap for compensation claimants. The burden of proof should be evaluated according to legal standards, and must not be confused with scientific notions of probability. Traditional legal rules regarding the admissibility of evidence may have to be adapted to better serve the process of adjudication by administrative tribunals when the medico-legal issues involved and the disparity in the means of the parties, lead to an imbalance in access to scientific and medical information. Judicial "neutrality» may in itself create a form of bias in a context where inequality between the opposing parties is intrinsic to the nature of the compensation system.

* Une version précédente de ce texte a été présentée au $2^{\complement}$ colloque sur les maladies du travail au Québec, Sherbrooke, le 27 octobre 1991. L'auteure est consciente que les travailleuses aussi peuvent être atteintes de maladies professionnelles. L'utilisation du masculin n'a pas pour but de les exclure.

**. Avocate et professeure au Département des sciences juridiques, U.Q.A.M. 


\section{SOMMAIRE}

Introduction

1. Les différences entre le droit et la science . . . . . . . . . . 448

a) La gamme de réponses possibles ........... 448

b) Le sens du terme «probable» . . . . . . . . . . . 449

c) La notion de relation . . . . . . . . . . 451

2. L'examen de la preuve médicale . . . . . . . . . 456

a) Identifier le fardeau de preuve du réclamant . . . . . 456

b) Décrypter le langage et le raisonnement des médecins et des scientifiques $\ldots \ldots \ldots \ldots \ldots \ldots \ldots \ldots$

3. Trois problèmes: les études scientifiques déficientes, non produites en preuve ou inexistantes $\ldots \ldots \ldots \ldots \ldots \ldots 465$

a) Études déficientes $\ldots \ldots \ldots \ldots \ldots \ldots \ldots \ldots 4$

b) Études non produites en preuve $\ldots \ldots \ldots \ldots \ldots \ldots 466$

c) Études inexistantes ................. 469

Conclusion $\ldots \ldots \ldots \ldots \ldots \ldots \ldots \ldots \ldots \ldots \ldots \ldots \ldots \ldots \ldots$ 


\section{Introduction}

Les questions de nature médicale ou scientifique se présentent dans plusieurs contextes juridiques. Qu'on examine la responsabilité civile ou pénale du médecin ou qu'on se questionne sur l'aptitude de l'accusé à subir son procès, il demeure que ce sont des «oreilles de juristes» qui entendent et jugent des paroles de médecins. Bien que ce phénomène se présentait à l'occasion dans le cadre de procès soulevant des questions médico-légales, dans certaines nouvelles sphères de pratique les juristes sont confrontés quotidiennement à des questions exigeant une compréhension de données médicales.

Le nouveau ${ }^{1}$ contentieux dans le domaine de l'indemnisation des victimes de lésions professionnelles ou d'accidents de la route exige sinon une maîtrise tout au moins une bonne compréhension des questions médicales soulevées par la réclamation. Il va de soi que le procureur qui délègue à son témoin expert le soin de comprendre l'ensemble des aspects médicaux abdique ses responsabilités et risque de passer à côté des questions les plus importantes. Chaque lésion exige des connaissances particulières mais tous les dossiers qui soulèvent des questions médicales, qu'ils portent sur un accident ou sur une maladie professionnelle, exigent une compréhension de base de la médecine, de la science et du droit. Au-delà de la compréhension des termes médicaux, il faut avant tout examiner le sens particulier de certains termes généraux utilisés par les médecins et le type de raisonnement qui est propre à la science en général et à la médecine en particulier. Parallèlement à cette réflexion, il faut se rappeler des fonctions particulières du droit.

1. Lintervention répandue de juristes dans le domaine de l'indemnisation des lésions professionnelles date de la création du droit d'appel à la Commission des affaires sociales en 1977. Ce même tribunal entend les appels en matière d'indemnisation des lésions reliées aux accidents d'automobile depuis 1978. Aujourd'hui les bureaux de révision paritaire de la Commission de la santé et de la sécurité du travail et la Commission d'appel en matière de lésions professionnelles entendent également les causes portant sur les questions médico-légales. 


\section{Les différences entre le droit et la science}

a) La gamme de réponses possibles

La science prétend à la vérité. Le dictionnaire Petit Larousse définit le terme «science» ainsi: «Ensemble cohérent de connaissances relatives à certaines catégories de faits, d'objets ou de phénomènes obéissant à des lois et vérifiées par les méthodes expérimentales.... Discipline ayant pour objet l'étude des faits, des relations vérifiables». La médecine et les médecins s'identifient à la communauté scientifique et aux valeurs scientifiques.

Le scientifique et le médecin ont toujours trois choix de réponses à une question qui leur est posée: oui, non et on ne sait pas. La catégorie «on ne sait pas» est vaste, particulièrement dans le domaine de la santé au travail. Tout porte à croire que ceux qui contrôlent en bonne partie le financement des recherches scientifiques ont un certain intérêt à maintenir l'incertitude la plus grande ${ }^{2}$.

Contrairement à la science, le droit n'a pas la prétention de proclamer la vérité. Ceci ne veut pas dire que le droit ne valorise pas l'honnêteté. Cependant, le juge ne peut pas s'abstenir de décider sous prétexte que la réalité est incertaine. En droit, il n'y a que deux réponses possibles car le juge ne peut refuser de se prononcer parce qu'il ne sait pas.

Illustrons ce principe en l'appliquant à une réclamation pour une maladie professionnelle. Lorsque la Commission d'appel en matière de lésions professionnelles refuse de reconnaître une relation entre le travail et une maladie, elle affecte les droits du travailleur. Toutefois, le commissaire a le devoir de décider entre l'acceptation ou le refus de la demande du travailleur. Ce dernier doit convaincre le tribunal de la relation entre son travail et sa maladie. Le scientifique qui n'est pas certain de l'existence ou l'inexistence de la relation s'abstiendra de se prononcer, mais le commissaire placé dans la même situation n'a d'autre choix que de se prononcer sur la demande. Ainsi le défaut du scientifique d'affirmer l'existence d'une relation a le même effet

2. Voir: Samuel S. EPSTEIN, The Politics of Cancer, San Francisco, Sierra Club Books, 1979. 
juridique qu'une affirmation catégorique d'absence de relation. Dans les deux cas le travailleur assume seul les coûts de sa maladie.

Dans sa discipline, le scientifique s'abstient de se prononcer jusqu'au moment où il peut affirmer sa «vérité». En droit, le juge doit se prononcer sur les conséquences juridiques, même en l'absence de preuve de la «vérité». C'est pour cette raison que le droit détermine le degré de «vérité» nécessaire pour justifier sa conclusion. Ce degré de vérité variera en fonction des conséquences de sa décision. Si un justiciable doit être privé de sa liberté suite à la décision du juge, le droit exige une preuve hors de tout doute raisonnable avant la condamnation pénale de l'accusé. Si un justiciable réclame de l'argent en dédommagement, le droit donnera raison à celui dont la version est la plus probable. Le juge doit alors soupeser deux hypothèses et choisir la plus probable. Il juge selon la balance des probabilités c'est-àdire en fonction de la preuve prépondérante.

b) Le sens du terme «probable»

Les juristes qui exercent leur profession dans le domaine de l'indemnisation sont confrontés à un discours médical qui leur est souvent obscur et intimidant. Ils doivent se familiariser avec l'épicondylite, le tunnel carpien et la spondylolyse ankylosante. Les meilleurs d'entre eux étudient leurs dictionnaires médicaux et discutent longuement avec leurs experts pour comprendre ces termes. Mais bien plus mystificateurs et dangereux sont les termes qui ont un sens en médecine et un tout autre sens en droit. Lorsque le même terme est utilisé pour désigner deux réalités, les chances de confusion sont beaucoup plus grandes. En tant que juriste, on sait qu'on ne comprend pas le terme «épicondylite» mais on a l'illusion de comprendre hotre expert lorsqu'il nous dit qu'il n'y a pas de relation entre le travail et la maladie ou qu'il est possible mais non probable que le travail ait causé la maladie. Les termes «probable», «relation» ou même «lien de causalité» ont un sens en droit, un autre sens en médecine et un troisième dans le langage ordinaire. $\mathrm{Si}$ ceux qui utilisent ces termes ne sont pas conscients de cette multiplicité de sens, il y a un danger réel que des malentendus surviennent.

Lorsque, dans le cadre d'une réclamation pour une maladie professionnelle, un juriste interroge un témoin médical sur la probabilité de l'existence 
d'une relation entre la maladie et le travail, la question qu'il formule présuppose une réponse affirmative dans l'éventualité où il est certain à plus de $50 \%$ de l'existence de la relation. Le juriste veut savoir si la probabilité que le travail ait contribué à la maladie est plus grande que la probabilité qu'il n'y ait pas contribué. Sauf exception, le témoin médical ou scientifique répondra à la question en appliquant un degré de probabilité beaucoup plus élevé. Il lui faudra parfois être certain à $95 \%$ de l'existence d'une relation avant d'affirmer qu'il est probable qu'une relation entre le travail et la maladie existe. En médecine, la certitude nécessaire pour confirmer l'incidence étiologique est élevée car dans ce domaine, une telle affirmation implique souvent une intervention chirurgicale ou un plan de traitement. Une erreur pourrait entraîner des conséquences désastreuses. Selon les exigences de la formation scientifique ou médicale, le degré de probabilité se situe plutôt à $95 \%$ et parfois même à $99 \%$ mais les médecins, et encore davantage les scientifiques, ont toujours la possibilité de l'abstention. Le juge, lui, n'a pas cette option. Lorsqu'il conclut à l'ignorance à l'égard de la relation, le travailleur ou l'accidenté en assume les conséquences. Lorsque la vérité ne peut être atteinte le juge a l'obligation de choisir, des deux versions, celle qui lui paraît la plus plausible. Lorsque les médecins ou scientifiques qui témoignent ne parlent que de possibilités ou lorsqu'ils refusent d'affirmer qu'une relation est probable, le juge interprétera leur réticence comme étant une affirmation qu'il y a moins de $50 \%$ de chances qu'il y ait une relation entre le travail et la maladie. Il est donc du devoir du procureur de bien formuler ses questions. Dans le cadre d'une réclamation pour une maladie professionnelle, le droit cherche à déterminer s'il est plus probable que non qu'un lien de causalité existe entre le travail et la maladie.

La Cour suprême du Canada vient de rappeler cette distinction entre la notion de probabilité en droit et en médecine dans l'affaire Snell $c$. Farrell ${ }^{3}$. On peut y lire le passage suivant:

«Le fardeau ultime de la preuve incombe au demandeur, mais en l'absence de preuve contraire présentée par le défendeur, une inférence de causalité peut être faite même si une preuve positive ou scientifique de la causalité n'a pas été produite [...] Par conséquent,

3.

[1990] 2 R.C.S. 311, 330. 
il n'est pas essentiel que les experts médicaux donnent un avis ferme à l'appui de la théorie de la causalité du demandeur. Les experts médicaux déterminent habituellement l'existence de causalité en des termes de certitude, alors qu'une norme inférieure est exigée par le droit. Comme l'a souligné Louisell dans Medical Malpractice, vol. 3, l'expression [TRADUCTION] "à votre avis, avec un degré raisonnable de certitude médicale" qui constitue la forme de question normalement posée à un expert médical, est souvent mal comprise. L'auteur explique à la p. 25-57 [TRADUCTION]: "Un grand nombre de médecins ne comprennent pas l'expression [...] car ils parlent habituellement de 'certitudes' à 100 pour 100 , alors que les certitudes 'raisonnables' requises en droit exigent seulement une probabilité supérieure, c'est- à-dire à 51 pour 100 ."»

La pertinence de cet arrêt dans le domaine des lésions professionnelles a été soulignée par la Commission d'appel en matière de lésions professionnelles dans une décision portant sur le syndrome du tunnel carpien. Le Bureau de révision paritaire avait refusé la réclamation au motif que le témoin expert avait qualifié de «possible» la relation entre le travail et la lésion. Le commissaire siégeant en appel de cette décision souligne la différence entre la probabilité médicale et la probabilité juridique pour conclure qu'en l'espèce la preuve prépondérante justifiait l'indemnisation ${ }^{4}$.

c) La notion de relation

Même si les intervenants font l'effort d'uniformiser leur langage dans le sens suggéré par la Cour suprême en ce qui concerne la probabilité d'une relation, une deuxième source de confusion de langage se produit dans la détermination de la nature de la relation exigée pour justifier l'indemnisation. Doit-on prouver que le travail (ou un accident) a causé la lésion ou suffit-il que le travail ait contribué au développement de la maladie? Suffit-il de constater que la lésion ne se serait pas développée n'eût été du travail? Comment doit-on départager la «responsabilité» de maladies à étiologies multiples telles que la plupart des maux de dos et des maladies cardio-vasculaires?

4. Martin et Brasserie Molson O'Keefe Ltée, C.A.L.P. $n^{\circ} 12779-62-8905,28$ janvier 1992. 
De temps à autre la Commission d'appel en matière de lésions professionnelles nous rappelle qu'il n'est pas nécessaire d'avoir une preuve hors de tout doute raisonnable sur la question de la relation entre la maladie et le travail ${ }^{5}$, mais rares sont les décisions où elle s'attarde au sens à donner au fardeau de preuve ${ }^{6}$. Bien que tous s'entendent sur la nécessité d'un lien de causalité entre maladie et travail, le degré de «responsabilité» du travail n'en est pas un de causalité exclusive. La loi exige que l'accident ait «entraîné» une lésion ${ }^{7}$. La jurisprudence de la Commission d'appel en matière de lésions professionnelles qui est fidèle aux principes de la responsabilité civile, estime qu'il suffit que l'accident du travail ou la maladie professionnelle $^{8}$ ait rendu symptomatique une lésion personnelle jusqu'alors asymptomatique pour justifier l'indemnisation de l'incapacité. Lorsque l'accident rend invalidant un état qui ne l'était pas auparavant, il y a un lien de causalité entre l'accident du travail et les limitations fonctionnelles qui justifie l'indemnisation $^{9}$ et cela même si le travailleur était plus vulnérable que ses collègues. Celui qui indemnise «assume les risques inhérents à la qualité et à la personnalité de sa victime» ${ }^{10}$. L'obésité ${ }^{11}$, la faible constitution ${ }^{12}$, l'arthro$\mathrm{se}^{13}$, la personnalité nerveuse $\mathrm{e}^{14}$ et même la spondylolyse avec spondylolisthé$\operatorname{sis}^{15}$ constituent toutes des conditions personnelles qui ne justifient pas un

5. Voir à titre d'exemple: Giampetrone et Usinage de précision de Montréal, [1988] C.A.L.P. $\mathrm{n}^{\circ}$ 01246-60-8611.

6. Pour une excellente étude sur l'ensemble de cette question, voir: Diane L. DEMERS, «Les maladies professionnelles et la plausibilité biologique», [1991] 25 R.J.T. 29.

7. Loi sur les accidents du travail et les maladies professionnelles, L.R.Q., c. A-3.001, art. 2 où on définit le terme «accident du travail».

8. Produits chimiques Expro Inc. c. C.A.L.P., [1988] C.A.L.P. 187 (C.S.), en appel, $\mathrm{n}^{\circ}$ 50009-000306-886.

9. Lavoie et 2332-3827 Québec Inc., C.A.L.P. $\mathrm{n}^{\circ}$ 04124-63-8708.

10. Jean-Louis BAUDOUIN, La responsabilité civile délictuelle, $3^{c}$ édition, Cowansville, Éditions Yvon Blais, 1990, p. 118. L'auteur y confirme que la «Thin Skull Rule» de la Common Law reçoit son application en droit québécois.

11. Simmon's Ltée et Fournier, [1988] C.A.L.P. 673.

12. Labelle-Dagenais et Zeller's Ltée, [1988] C.A.L.P. 316.

13. Produits chimiques Expro Inc. c. C.A.L.P. et Richer, précitée, note 8; voir aussi: Collona et Maçonnerie G.E. Ltée et C.S.S.T., [1988] C.A.L.P. 288.

14. Gagnon et C.A.R.R.A., [1989] C.A.L.P. 769; Guitar-Prieur et Miracle Mart, [1989] C.A.L.P. 738.

15. Beaulieu et Société d'aluminium Reynolds (Canada) Ltée, C.A.L.P. n ${ }^{\circ} 10424-04-8812$, 17 mai 1991; Turbine et Howard Bienvenu, C.A.L.P. $n^{\circ}$ 17185-08-9002, 18 mars 1991. 
refus d'indemnisation si elles n'étaient pas problématiques ou invalidantes avant l'accident. Selon la logique juridique, la seule qui devrait régir le droit à l'indemnisation, il est donc clair qu'il n'est pas nécessaire d'avoir la preuve que le travail a été constitué la cause principale de la lésion pour accueillir une réclamation. L'indemnisation sera payable si le travail a été une cause contributive à la survenance d'un dommage qui ne se serait pas manifesté à ce moment n'eût été du travail.

Certains auteurs prétendent que la notion de relation particulière à un régime d'indemnisation sans faute est moins étroite que la relation exigible dans un contexte civil, du fait que le caractère «sans faute» du régime rend inutile l'exigence du caractère prévisible des dommages. Alors qu'en responsabilité civile l'intervention de fautes subséquentes peut rompre le caractère direct des dommages, les mêmes considérations ne devraient pas s'appliquer dans un régime sans faute ${ }^{16}$. Les juridictions canadiennes de Common Law se penchent soigneusement sur les questions de relation. Dans ces juridictions, on exige que le travail ait contribué au développement de la maladie. Parfois, on exige une contribution plus significative lorsqu'il s'agit d'une maladie à étiologie multiple ${ }^{17}$. Au Québec, il semblerait que la Commission d'appel en matière de lésions professionnelles préfère évaluer chaque dossier à la lumière de principes généraux sans s'attarder à une définition rigoureuse de la notion de relation. Il n'en demeure pas moins que les principes de base, conformes à la lettre et à l'esprit de la législation, semblent n'exiger qu'une démonstration de la contribution du travail au développement de la lésion. Ainsi seront exclues les lésions qui ne constituent

16. Le Doyen Arthur Larson, qui est l'autorité principale en matière de droit de l'indemnisation des lésions professionnelles aux États-Unis est de cet avis. Voir notamment: Arthur LARSON, "The Legal Aspects of Causation in Workmen's Compensation", (1954) 8 Rutgers L. Rev. 423 - 426.

17. Voir Terence G. ISON, Workers' Compensation in Canada, 2 ed., Toronto, Butterworths, 1989 , p. 58 et suiv. Beaucoup de controverses entourent la définition du degré de relation nécessaire à la reconnaissance d'une lésion en droit ontarien. On peut comparer la Décision 918, [1988] 9 W.C.A.T.R. 48 avec la Décision 1018, [1989] 10 W.C.A.T.R. 82 où le Tribunal d'appel cherche à définir la relation exigée lorsque la réclamation porte sur une incapacité psychologique qu'on allègue être en relation avec le stress du travail. Pour une discussion de la jurisprudence ontarienne sur cette question voir Katherine IIPPEL, «Compensation for Mental - Mental Claims Under Canadian Law», (1990) 8 Behavioral Sciences \& The Law 375-398. 
que le développement normal de la condition personnelle. Lorsque les conditions de travail précipitent la manifestation d'une incapacité, celle-ci est indemnisable ${ }^{18}$.

Alors que la notion juridique de lien de causalité est confuse, l'intervention des médecins, avec leurs propres notions d'étiologie ou de relation, rend encore plus complexe une tâche déjà difficile. Lorsqu'on demande à un médecin si la lésion est en relation avec le travail, sans encadrer notre question de paramètres qualificatifs, il répond à une question sur l'étiologie: le travail a-t-il causé la maladie? Une affaire récente permet d'illustrer cette affirmation. Une technicienne de laboratoire est décédée des suites d'une méningite. Il était en preuve qu'elle avait, dans le cadre de son travail, manipulé des échantillons contaminés servant à diagnostiquer cette maladie. Interrogé sur le lien entre le travail et la maladie, un médecin a répondu qu'il n'était pas en mesure d'identifier l'origine de la contamination. Il a affirmé que «la technologie de notre époque ne nous permettra pas de prouver que c'est la souche du sujet index qui a été transmise à la technicienne». Il a quand même prétendu qu'il s'agissait d'une infection professionnelle, mais la C.S.S.T. a refusé la réclamation au motif qu'il n'était pas prouvé que le décès de la technicienne était relié à des risques particuliers de son travail $^{19}$. Pourtant, en droit, si on estime qu'il est probable qu'elle ait été en contact avec des contaminants au travail (la preuve non contestée êtait à cet effet) et qu'il est probable qu'un contaminant peut infecter la personne qui le manipule (la technicienne est décédée 6 jours après avoir manipulé l'échantillon de la même bactérie et la période d'incubation était conforme aux connaissances scientifiques), on peut conclure par présomption de fait qu'il est probable que celle qui a été effectivement contaminée l'a été à l'occasion du travail, d'autant plus qu'aucune autre possibilité de contamination n'avait été mise en preuve. Le tribunal ou l'organisme administratif a pour rôle de

18. Lefebvre c. Commission des affaires sociales du Québec, [1991] R.J.Q. 1864 (C.A.), jugement du juge Vallerand; voir aussi: Antenucci c. Canada Steamship Lines Inc. et C.S.S.T., [1991] R.J.Q. 968 (C.A.). Ces deux décisions de la Cour d'appel du Québec démontrent la confusion qui persiste dans le droit québécois entre la notion d'accident du travail et la notion de relation entre une lésion et le travail.

19. Décision d'une agente d'indemnisation dans le dossier $\mathrm{n}^{\circ} 10169580,21$ décembre 1991. 
déterminer la meilleure hypothèse quant à la cause de la maladie dans un cas particulier $^{20}$.

À chaque fois qu'un médecin, qu'il soit expert, arbitre, ou traitant, déclare qu'une lésion devrait ou ne devrait pas être indemnisée, il donne une opinion juridique. Très souvent les médecins fonctionnaires de la C.S.S.T. ou de la S.A.A.Q. affirment qu'une réclamation ne devrait pas être reconnue, non pas parce que le travailleur n'était pas malade mais parce que la loi ne couvre pas son cas, la plupart du temps parce que le travailleur souffrait d'une condition préexistante. Certains médecins sont d'opinion que la C.S.S.T. ne devrait jamais verser d'indemnisation lorsque le travailleur était prédisposé à la lésion. D'autres estiment qu'un infarctus ou une dépression ne devrait pas être indemnisable à cause de la difficulté à déterminer l'étiologie de ces maladies. Même lorsque la délimitation entre le droit et la médecine est clairement énoncée, les témoins médicaux sont parfois réticents à affirmer que le travail a pu aggraver la condition personnelle du travailleur parce qu'ils estiment injuste que les employeurs assument le coût de l'ensemble de l'incapacité alors qu'ils ne sont «responsables» que d'une partie ${ }^{21}$.

Le rôle des médecins est de témoigner sur des faits. Ils ne sont pas là pour émettre des opinions quant à l'opportunité de reconnaître certains types

20. T.G. ISON, op. cit., note 17, p. 51: «... The legally relevant question [is] what is The best available hypothesis about the cause of the disease in the particular case».

21. Ce point fut éloquemment illustré par F.S. MILNER, «Heart Disease Due to Occupational Emotional Stress: A Compensable Claim Under Oregon Workers' Compensation Law?», [1979] 10 Environmental Law 159, à la page 176, note 63; qui rapporte le témoignage suivant d'un cardiologue lors de sa comparution au Cardiac Subcommittee of the Statutory Advisory Committee on Medical Care for the Oregon State Legislature: «As near as I can tell; the lawyer is primarily concerned with whether there is reasonable cause or suspicion that the work activity is a precipitating event or contributed in any way. It's the fault of the law that says that if there is a contribution there is a certain award. I think that in many instances we can say that there could have been some precipitation or aggravation of the chronic degenerative disease by some kind of work activity. The problem with that is the inequity in making the whole thing compensable». 
de lésions. Parfois les tribunaux vont même souligner qu'ils dépassent leur compétence en agissant ainsi ${ }^{22}$.

\section{L'examen de la preuve médicale}

Dans le cadre de l'interprétation de la preuve médicale et scientifique deux éléments doivent être démystifiés pour éviter des confusions découlant de l'interaction entre les différentes disciplines. Il faut bien identifier le fardeau de preuve du réclamant. Il faut ensuite décrypter le discours et le raisonnement scientifiques afin de les transformer en données susceptibles d'éclairer véritablement le tribunal ${ }^{23}$.

a) Identifier le fardeau de preuve du réclamant

En Ontario, le discours officiel des organismes chargés de déterminer le droit des travailleurs à une indemnisation veut que le travailleur n'ait pas de fardeau de preuve à supporter. Il appartient à la Commission des accidents du travail de l'Ontario d'enquêter pour déterminer si oui ou non il a le droit d'être indemnisé ${ }^{24}$. Au Québec le régime d'indemnisation est marqué

22. Voir la décision non publiée de la Commission des affaires sociales, du 25 juillet 1989, dans le dossier $\mathrm{n}^{\circ} \mathrm{AT}-58031$, où le témoin expert, psychiatre, avait émis l'opinion suivante: «Nous ne pensons pas que cette réaction anxio-dépressive s'inscrive dans le cadre de la C.S.S.T. et qu'il y aurait danger à vouloir médicaliser ou psychiatriser ce genre de situation». La Commission des affaires sociales estime «qu'il excède le champ de compétence de ce médecin, compétence qui se limite aux aspects médicaux du problème plutôt qu'à ses aspects légaux ou sociologiques».

23. Évidemment il ne s'agit pas ici d'imposer en termes absolus l'hégémonie des valeurs juridiques sur les valeurs scientifiques. Par contre, dans un contexte de détermination des droits des parties à qui une législation statutaire est applicable, la règle de droit doit toujours primer sur les valeurs élaborées dans d'autres disciplines ayant des finalités distinctes. Lorsqu'on est dans un contexte d'adjudication, le discours de l'ensemble des disciplines qui peuvent contribuer à la preuve doit être filtré de manière à être interprété et appliqué selon des normes juridiques.

24. Voir T.G. ISON, op. cit., note 17, p. 206. L'auteur souligne qu'en pratique la Commission des accidents du travail agit à l'occasion comme si le travailleur avait un fardeau de preuve mais conclut que le fardeau de preuve doit être assumé par l'organisme décisionnel et non pas par les parties. Cette approche découle du caractère social de la législation. Historiquement la création des Commissions des accidents du travail a toujours été justifiée par la nécessité de faciliter l'accès à l'indemnisation pour le travailleur en 
aujourd'hui par ce vestige du régime de responsabilité civile que constitue le fardeau de preuve. Dans tous les cas, il appartient à celui qui cherche à se faire indemniser de démontrer par une prépondérance de preuve qu'il a droit aux prestations. Parfois le législateur l'aide dans l'élaboration de sa preuve en adoptant des présomptions telles que celles prévues aux articles 28,29 et 95 de la Loi sur les accidents du travail et les maladies professionnelles ${ }^{25}$. Même lorsqu'une présomption légale peut être invoquée, le travailleur a le fardeau de démontrer qu'il satisfait aux conditions d'application de la présomption et il doit le démontrer au moyen d'une preuve prépondérante.

Illustrons cet énoncé par l'exemple du travailleur victime de l'amiantose qui a travaillé dans une mine d'amiante. L'article 29 de la loi nous réfère à l'annexe relative aux maladies professionnelles. On y apprend que celui qui souffre d'amiantose et qui peut démontrer qu'il a été exposé à l'amiante est présumé avoir contracté la maladie par le fait ou à l'occasion du travail. On présumera également que cette maladie est caractéristique du travail ou reliée aux risques particuliers du travail. Grâce à cette présomption, le fardeau de preuve du travailleur est moindre que celui qu'il aurait dû supporter si la maladie ne faisait pas partie de la liste mais le travailleur a quand même un fardeau important. Il doit démontrer selon la prépondérance des probabilités qu'il est atteint de l'amiantose et, toujours selon la prépondérance des probabilités, qu'il a été exposé à l'amiante à son travail. S'il ne réussit pas à démontrer cela, la présomption de l'article 29 ne s'applique pas.

Lorsque le travailleur est atteint d'une maladie qui n'est pas énumérée à l'annexe, il doit assumer un fardeau de preuve qui comporte un plus grand nombre d'éléments. Cependant le degré de probabilité appliqué dans l'évaluation de chaque élément demeure celui de la prépondérance. Sans l'aide de la présomption statutaire le travailleur doit établir quatre éléments pour justifier sa réclamation en vertu de l'article 30 de la Loi sur les accidents du travail et les maladies professionnelles. Il doit démontrer qu'il est atteint d'une maladie, contractée par le fait ou à l'occasion du travail, que la maladie dont il est

déjudiciarisant le processus de réclamation. Voir aussi: Katherine LIPPEL, Le droit des accidentés du travail à une indemnité: analyse historique et critique, Montréal, Les Éditions Thémis Inc., 1986.

25. Précitée, note 7 . 
atteint est par ailleurs caractéristique de ce travail ou reliée aux risques particuliers du travail et enfin, que cette maladie a entraîné une incapacité ou un dommage. Illustrons cet énoncé par l'exemple d'un cuviste exposé au goudron et atteint du cancer de la vessie. Afin de faire reconnaître sa maladie en vertu de l'article 30 de la Loi sur les accidents du travail et les maladies professionnelles, ce travailleur doit démontrer par une preuve prépondérante:

1. qu'il est atteint du cancer de la vessie,

2. qu'il a été exposé au goudron à son travail,

3. qu'il a contracté le cancer par le fait ou à l'occasion du travail, et

4. que le cancer de la vessie est caractéristique d'un travail impliquant une exposition au goudron ou relié aux risques particuliers de celui-ci.

Pour chacun de ces éléments la version du travailleur doit être plus probable que la version contraire. Ceci implique que le travailleur peut être indemnisé même lorsque le diagnostic est incertain ${ }^{26}$ si le médecin est d'opinion qu'il est probablement atteint du cancer de la vessie. On n'exigera pas que le travailleur démontre hors de tout doute raisonnable qu'il y avait du goudron dans ses lieux du travail, de la même manière qu'on n'exigerait pas une preuve hors de tout doute raisonnable du harcèlement sexuel ou d'un trouble de voisinage qu'on allègue être en relation avec un dommage. Il suffit que le décideur soit convaincu par une preuve prépondérante que la nuisance était réelle. Dans son raisonnement le juriste exige que chaque élément du fardeau de preuve, pris isolément, soit démontré par une preuve prépondérante $(50 \%+1$ de certitude). Par contre le médecin ou le scientifique serait plutôt porté à examiner la question dans son ensemble et en ce faisant, il diminuerait radicalement les chances de réussite du travailleur.

26. En droit civil, le dommage doit être «certain», mais dans J.-L. BAUDOUIN, op. cit., note 10, p. $108, n^{\circ} 180, l^{\prime}$ auteur définit un préjudice «incertain» comme un préjudice «qui ne se réalisera probablement pas». Il ajoute que «[l]es tribunaux n'exigent pas, en effet, une certitude absolue mais une simple probabilite». On doit conclure qu'en droit «certain» peut vouloir dire «probable», alors qu'en médecine «probable» peut vouloir dire «certain». De toute façon le «dommage» dans le contexte de l'indemnisation est lincapacité et non pas le diagnostic comme tel. La «certitude» des dommages dans les régimes sans faute est assurée par la structure même du régime, qui permet aux organismes décisionnels de mettre fin aux prestations lorsque le bénéficiaire redevient apte au travail. 
L'importance de cet imbroglio médico-légal a été éloquemment illustrée par le Professeur Ison dans une étude préparée par l'organisme gouvernemental ontarien chargée de déterminer les critères de reconnaissance des maladies professionnelles. En appliquant ses affirmations à notre exemple, nous examinerons les questions en litige:

1. Il est atteint du cancer de la vessie. Il faut $50 \%+1$ de chances qu'il souffre de cette maladie. S'il est malade et qu'il souffre probablement du cancer de la vessie, cet élément du fardeau de preuve est établi même si les médecins ne sont pas certains de leur diagnostic (51\% de certitude, donc preuve prépondérante établie).

2. Il a été exposé au goudron à son travail. On doit se demander s'il est plus probable que son travail l'ait exposé à une cancérigène tel le goudron pour des périodes et à un niveau qui auraient pu contribuer au développement du cancer de la vessie (51\% de certitude, donc preuve prépondérante établie).

3. Il a contracté le cancer par le fait ou à l'occasion de son travail. Est-il plus probable que non que la maladie a été contractée au travail ou que le travail a contribué au développement de la maladie? (51\% de certitude, donc preuve prépondérante établie).

4. Le cancer de la vessie est caractéristique du travail de cuviste ou relié aux risques particuliers d'un travail comportant une exposition au goudron: la littérature ou les scientifiques qui témoignent confirment-ils qu'il y a plus de $50 \%$ de chances que le cancer de la vessie soit relié à l'exposition au goudron $(51 \%$ de certitude, donc preuve prépondérante établie).

Si chaque élément de son fardeau est démontré par une preuve égale ou supérieure à $50 \%+1$, le travailleur a rencontré les exigences de la loi et a le droit d'être indemnisé.

Pourtant lorsqu'on demande à l'expert si le travailleur est atteint d'une maladie professionnelle sans décortiquer la question, on doit s'attendre à une réponse négative, car le scientifique, sans balises juridiques, répondra selon 
la probabilité globale de l'événement, calculée en fonction des formules mathématiques de probabilités d'événements cumulatifs en effectuant le calcul suivant: $51 \% \times 51 \% \times 51 \% \times 51 \%=6.76 \%$. Statistiquement la probabilité que l'ensemble des prémisses soient simultanément vraies, abstraction faite du contenu des prémisses, est mince. Le doute sur le diagnostic multiplié par le doute sur l'exposition au contaminant crée, mathématiquement parlant, un doute beaucoup plus grand quant à la réalisation simultanée des deux prémisses. En droit, il faut regarder chaque question en litige séparément et déterminer, élément par élément, si le travailleur s'est déchargé de son fardeau de preuve. En procédant ainsi le travailleur dans notre exemple s'est déchargé de son fardeau de preuve et il devrait être indemnisé ${ }^{27}$.

b) Décrypter le langage et le raisonnement des médecins et des scientifiques

Nous avons vu que la double déformation du langage juridique et du langage médical permet d'affirmer que dans certaines circonstances le terme «probable» en médecine peut vouloir dire «certain» alors qu'en droit le terme «certain» peut vouloir dire «probable». Il n'est donc pas surprenant de constater qu'une certaine confusion puisse survenir lorsque les deux discours se rencontrent et que cette confusion ne peut être qu'exacerbée par le fait que chacun a l'illusion de bien comprendre l'autre. Le processus adjudicatif est suivi non seulement par les médecins et les juristes mais également par les justiciables et les représentants des parties qui ne sont pas juristes. Voilà une raison de plus pour affirmer que l'apparence de justice est susceptible de disparaître. Trois discours se fréquentent: le discours médical, le discours

27. Cet exemple est tiré de Terence G. ISON, Compensation for Industrial Disease Under The Workers' Compensation Act of Ontario, Comité des normes en matière de maladies professionnelles, septembre 1989 , p. 10 . Bien que l'auteur se réfère à la législation ontarienne on peut conclure que la législation québécoise invite une interprétation analogue. Nous croyons que l'exemple présente certains défauts sur le plan mathématique en ce sens que le Professeur Ison ne tient pas compte du fait que les quatre variables dans le calcul ne sont pas indépendantes les uns des autres, mais le principe qu'il illustre est éloquent et, à notre sens, applicable au droit québécois. Pour les juristes qui voudraient approfondir les éléments de base de calculs des probabilités au sens mathématique on peut consulter Donald H. SANDERS, Franklin A. MURPH et Robert J. ENG, Les statistiques: une approche nouvelle, Montréal, McGraw-Hill, 1984, pp. 102 et suiv. 
juridique et le bon vieux langage du dictionnaire. Un même mot, comme le mot «certain» peut avoir trois sens différents, selon l'interlocuteur.

Ce phénomène peut sembler banal. Tous peuvent être victime d'une telle ambiguïté. Cependant, dans un contexte d'indemnisation, l'imbroglio a un effet négatif systémique sur les droits des réclamants et des demandeurs. Il est du devoir du représentant du demandeur de s'assurer que l'ensemble des interlocuteurs se comprennent et que le langage et le raisonnement retenus pour les fins décisionnelles soient ceux du droit. Pour ce faire, le procureur du demandeur a un double rôle à jouer: celui d'outiller ses témoins experts afin qu'ils puissent répondre à des questions juridiquement appropriées sans craindre d'être critiqué sur le plan scientifique de la part de leurs pairs et celui de démystifier le langage des experts de la partie adverse afin de s'assurer que le tribunal ne soit pas induit en erreur.

En préparant la preuve de la demande, il faut bien circonscrire l'objectif de l'expertise et s'entendre avec l'expert sur les balises juridiques applicables. Si l'expert estime qu'une relation possible existe, il faut tenter de clarifier le sens qu'il attribue au terme «possible» et poser les questions clairement afin que ses réponses respectent à la fois le langage et le fardeau juridiques de même que l'intégrité scientifique.

La partie défenderesse, qu'il s'agisse de l'employeur, des organismes administratifs qui refusent une réclamation ou d'un défendeur en responsabilité civile, a intérêt à ce que ses témoins s'expriment en termes de certitudes scientifiques. Il est du devoir du Tribunal de bien vérifier que le médecin qui infirme une relation veut dire qu'il est improbable qu'une relation existe. S'il veut simplement dire qu'il n'a pas assez d'éléments pour avoir la conviction scientifique mais qu'il y a plus que $50 \%$ de chances que la relation existe, la relation doit être inférée. Il est inutile d'essayer de forcer le médecin à changer de discours mais il est primordial qu'il balise sa notion de «probabilité» et de «certitude» afin que les décideurs puissent tirer les conséquences juridiques de son opinion d'expert.

Les particularités du raisonnement scientifique doivent également être démystifiées. Les experts se fient souvent sur les études épidémiologiques pour confirmer ou infirmer une relation. Parfois ils se basent sur des études 
qui démontrent que statistiquement une relation peut s'inférer à partir d'un nombre donné d'années d'exposition ou à partir d'une exposition à un certain niveau de concentration d'un contaminant. Ces études visent à démontrer statistiquement les risques de contracter une maladie suite à l'exposition à un contaminant. Aucune étude de ce genre ne vise à déterminer si le cancer du travailleur « $\mathrm{X}$ » est en relation avec son travail à l'usine «y». Pour sa part, le droit ne cherche pas à valider des expériences scientifiques, il cherche à déterminer le sort d'un travailleur en particulier.

Dans le cadre d'une demande d'indemnisation il faut adapter les notions scientifiques de probabilités au contexte juridique. La distinction entre les objectifs de la science et ceux du droit apparaît bien de l'analyse suivante:

«La balance des probabilités en droit, c'est la conviction acquise à partir d'un examen de la preuve particulière à un litige. Dans un procès en responsabilité civile, il ne s'agit pas de savoir ce qui se produit généralement, ou le plus souvent, quand certaines conditions sont réunies. Il s'agit plutôt de déterminer ce qui s'est produit dans ce cas, à partir de l'ensemble de la preuve présentée au juge. En d'autres termes, pour reprendre une analogie particulièrement heureuse, il ne s'agit pas de savoir quelles sont les chances qu'un numéro soit stiré à la loterie, mais plutôt de savoir qui a gagné. La preuve statistique envisagée seule est tout aussi inutile pour décider de la chaîne causale en droit qu'elle ne l'est pour nous apprendre quel a été, dans les faits, le numéro gagnant ${ }^{28}$.

Parmi les erreurs qui résultent de la confusion entre ces deux fonctions on doit souligner l'utilisation des calculs de risques pour déterminer la probabilité qu'un travailleur en particulier soit malade à cause du travail. Lorsque les décideurs exigent la preuve d'un nombre prédéterminé d'années d'exposition avant de reconnaître une relation entre un contaminant et une maladie, ils confondent la notion de risque avec celle de probabilité. Parfois, en se basant sur une étude scientifique; les décideurs reconnaissent qu'un contaminant peut causer la lésion mais refusent la réclamation au motif qu'une.

28. Extrait de Daniel JUTRAS, «Expertise scientifique et causalité», communication livrée au Congrès du Barreau du Québec, juin 1991, pp. 27-28. Cité avec la permission de l'auteur. 
exposition de quinze ans est insuffisante, la formule mathématique de probabilité exigeant une exposition de 19 ans. On oublie dans ces cas que le nombre d'années d'exposition visé dans une telle formule représente une moyenne, et que pour y arriver, il faut que certains travailleurs aient été exposés moins longtemps et d'autres plus longtemps. Si les décideurs exigent un nombre précis d'années d'exposition, ils écartent automatiquement des réclamations légitimes provenant de travailleurs plus vulnérables que la moyenne. L'absurdité de ce type de raisonnement devient plus évidente lorsqu'on l'applique à des situations relativement courantes. Le risque de subir un accident d'automobile mortel à $30 \mathrm{~km}$ à l'heure est peut être de $.01 \%$ mais si votre client est décédé dans un tel accident d'automobile, ce chiffre ne peut pas servir à convaincre le tribunal qu'il n'est pas décédé à cause de l'accident d'automobile $^{29}$. Une fois que l'événement se réalise, le taux de risque ne peut servir pour mesurer la probabilité de la relation. Pourtant les tribunaux administratifs appliquent parfois un tel raisonnement ${ }^{30}$.

Une décision du Bureau de révision paritaire de la C S.S.T. illustre bien ce point. Dans cette affaire, un travailleur demandait à être indemnisé pour un cancer de la vessie qu'il allèguait être en relation avec son travail dans une fonderie où il avait été exposé à plusieurs contaminants, et notamment au goudron volatile. Après l'analyse d'une étude scientifique sur le sujet le Bureau de révision paritaire conclut ainsi: «De cette étude scientifique, le Bureau de révision paritaire constate que le seuil ou le niveau acceptable des risques qui permet d'établir une relation entre une exposition professionnelle aux émanations de goudron volatiles et l'apparition d'un cancer de la vessie est de 19 années goudron» ${ }^{31}$. Il procède à analyser les années de travail du réclamant et conclut au rejet de la réclamation parce qu'il n'a que 17.48 années goudron. On doit s'interroger sur le sens des termes «niveau acceptable des risques» dans ce contexte. Un risque acceptable normalement qualifie un seuil en bas duquel un contaminant ne présente pas de danger. Utiliser le

29. Exemple fourni par Mme Diane Demers dans le cadre de la discussion qui a suivi la présentation de notre communication au 2 colloque sur Les maladies du travail au Québec, Sherbrooke, le 27 octobre 1991.

30. Pour une critique de l'utilisation de critères de reconnaissance prédéterminés voir: T.G. ISON, op. cit., note 17.

31. Simard c. S.E.C.A.L. B.R.P. n 60201854,3 octobre 1989, p. 9. L'assesseur syndical était dissident. 
terme dans le contexte où le travailleur est atteint du cancer après avoir été exposé pendant 17.48 années à un produit que l'étude reconnaît comme étant cancérigène nous force à conclure que le tribunal cherche à qualifier le niveau de risque exigé pour avoir une certitude raisonnable de ne pas indemniser un travailleur inutilement. Il ne s'agit pas d'un contexte normatif où l'objectif est de déterminer le niveau d'exposition acceptable. Le calcul mathématique vise à déterminer la probabilité pour les fins de l'indemnisation ${ }^{32}$. Le travailleur voulait savoir si son cancer de la vessie était indemnisable, puisqu'il a travaillé 17.48 années avec le goudron volatile, les B.P.C. et d'autres produits de même nature. Dans la décision, on lui répond qu'il a effectivement le cancer de la vessie, que ce type de cancer est caractéristique du travail impliquant une exposition à ces produits, mais que, dans son cas, il n'a pas réussi à prouver qu'il avait contracté son cancer à son travail, n'ayant pas été exposé au produit pour une période de dix-neuf ans. On doit se demander si les décideurs estimaient qu'il était plus probable qu'il a contracté son cancer à cause de contaminants non identifiés auxquels il aurait été exposé en dehors de son travail. Il nous semble difficile d'accepter que la relation entre le travail et la maladie n'ait pas pu être reconnue par présomption de fait. Lorsqu'un travailleur est atteint d'une lésion de cette nature alors qu'il n'a pas été exposé au contaminant le nombre «requis» d'années d'exposition, il a démontré qu'il est plus vulnérable que le travailleur moyen. Il devrait néanmoins avoir droit à l'indemnisation, sa vulnérabilité n'étant pas un motif de refus même dans le domaine des maladies professionnelles ${ }^{33}$.

Les organismes décideurs ne se questionnent pas souvent sur le rapport qui existe entre le risque que présente un produit et les conséquences pour la santé d'un travailleur en particulier. Le risque que présente une substance se mesure en comparant le taux de fréquence d'une maladie dans une population exposée à la substance, à celui d'une population non exposée. On conclut que le risque est plus élevé lorsque des études épidémiologiques démontrent qu'une maladie est présente plus fréquemment chez les gens exposés à une substance donnée. Plus le taux est élevé, plus le risque relié à la substance est grand. Même les études épidémiologiques les plus concluantes ne démontrent

32. Dans la décision, on fournit une telle formule mathématique, bien que les scientifiques spécifient qu'il appartient à l'administration de déterminer le niveau de probabilité exigé.

33. Produits chimiques Expro Inc. c. C.A.L.P. et Richer, précitée, note 8. 
pas la relation entre la maladie d'un travailleur et son travail. La Commission d'appel en matière de lésions professionnelles a reconnu que plus un risque est élevé, plus la probabilité de la relation entre la maladie de l'individu et son travail est grande ${ }^{34}$.

\section{Trois problèmes: les études scientifiques déficientes, non produites en preuve ou inexistantes}

a) Études déficientes

Il n'y a pas de doute que les témoins experts peuvent produire des études scientifiques à l'appui de leurs prétentions dans le cadre de leur témoignage ou en annexe à leur expertise. Il y a lieu de les étudier avec prudence afin de bien comprendre la méthodologie utilisée et sa pertinence pour les questions en litige. Lorsque l'auteur de l'étude n'est pas présent, et qu'il est difficile de déterminer les méthodes qu'il a utilisées, la fiabilité de l'étude peut être remise en question. Il n'est pas difficile de conclure que des rats ne sont pas morts du cancer à la suite de leur exposition à un produit si le taux d'exposition est tellement élevé qu'ils meurent d'intoxication aiguë avant d'avoir la «chance» de développer un cancer ${ }^{35}$. Lorsque les méthodes utilisées ne peuvent pas être déterminées, la Commission d'appel en matière de lésions professionnelles refusera parfois ${ }^{36}$ d'accorder foi à l'étude. Il est important de s'assurer que l'expert qui produit l'étude est en mesure de fournir non seulement l'extrait pertinent mais l'étude au complet. Par contre, un expert qui témoigne en faisant la synthèse de la littérature médicale jouira d'une grande crédibilité, particulièrement lorsqu'il produit en preuve les études sur lesquelles il s'appuie ${ }^{37}$.

34. Rinaldi c. Tootique Inc. et C.S.S.T., [1989] C.A.L.P. 416.

35. Exemple tiré de S. EPSTEIN, op. cit., note 2.

36. Mimeault et Les Constructions Morial Ltée, C.A.L.P. $n^{\circ}$ 01679-60-8612, 10 mai 1988 où la C.A.L.P. refuse d'accorder foi à une étude épidémiologique dont elle n'a reçu que des extraits. D'autres décisions ont accepté de considérer des résumés de communications, voir: J.M. Asbestos Inc. c. Wilson, [1990] C.A.L.P. 747.

37. Voir à titre d'exemple: De Charette et Les Entreprises M.M. Inc., C.A.L.P. n ${ }^{\circ}$ 02521-048703, 7 avril 1988; Rinaldi c. Tootique Inc., précitée, note 34. 
La Commission d'appel en matière de lésions professionnelles semble parfois tirer des conclusions qui ne se justifient pas nécessairement ${ }^{38}$. Une compréhension de l'ensemble des facteurs présents dans l'étude épidémiologique est essentielle. Si ces facteurs ne peuvent pas être connus on peut remettre en question la crédibilité de l'étude.

\section{b) Études non produites en preuve}

Qu'arrive-t-il lorsqu'une étude scientifique existe, à la connaissance de l'assesseur médical ou du commissaire de la Commission d'appel en matière de lésions professionnelles, mais qu'elle n'est pas en preuve? On serait porté à répondre de façon strictement légaliste à cette question et à conclure qu'aucune étude ou document scientifique qui n'a pas été produit en preuve ne peut être considérée $e^{39}$. Avant de répondre à cette question, il est opportun de réfléchir aux différentes circonstances qui sont susceptibles de se présenter.

Parfois l'assesseur médical a son opinion bien établie sur une question, opinion basée sur sa formation et sur les études analysées dans le cadre de cette formation. Parfois un commissaire est familier avec une nouvelle étude épidémiologique, versée en preuve dans un autre dossier et pertinente aux faits de la cause, mais inconnue des parties ou de leurs représentants. On peut aussi imaginer que l'assesseur ou le commissaire, frustré de la mauvaise

38. Voir par exemple: J.M. Asbestos Inc. c. Willson, précitée, note 36, où on s'appuie sur un résumé d'étude scientifique qui n'a pas démontré une relation statistiquement significative entre l'exposition à des faibles taux d'amiante et le développement de la maladie pour conclure, à l'encontre de plusieurs experts, qu'il n'était pas dangereux de retourner un amiantosé à son travail. Devant l'objection d'un témoin médical qui soulignait que les personnes faisant l'objet de l'étude n'étaient pas amiantosées, la Commission d'appel en matière de lésions professionnelles réplique, à la page 774 que «la cohorte de 447 travailleurs suivis annuellement étant tirée d'une population plus large de 1632 travailleurs exposés à l'amiante, il est fort probable que cette cohorte comprenait ceux qui présentaient des anomalies radiologiques initiales suggestives d'une amiantose précoce». Évidemment on ne saura jamais si les $13 \%$ des sujets ayant manifesté une détérioration était ceux qui présentaient justement de telles anomalies...

39. L'affaire Pfizer Co. Ltd. c. Sous-ministre du Revenu national, [1977] 1 R.C.S. 456 à la page 463 établit clairement que les décideurs d'un tribunal administratif n'ont pas le pouvoir de se rapporter à des renseignements qui «n'avaient pas été présentés en preuve et [auxquels] on n'avait pas renvoyé au cours de l'audience». 
qualité de la preuve ou de l'argumentation, entreprenne des recherches dans la littérature médicale pour élucider la situation factuelle.

S'opposer à toute prise en considération de données qui n'auraient pas été mises en preuve par les parties serait regrettable dans un contexte où le législateur a créé des tribunaux spécialisés justement dans le but de pallier au déséquilibre entre les parties et aux moyens insuffisants de ceux qui n'ont pas nécessairement la capacité de soutenir un débat sur des questions complexes de nature médicale ou scientifique. Un des plus grands obstacles que doivent affronter les travailleurs et les travailleuses victimes de maladies professionnelles provient du déséquilibre entre les parties. Lorsque la victime de la lésion n'est pas adéquatement représentée et que les études scientifiques disponibles qui appuieraient sa demande ne sont pas produites, il semble contraire à la philosophie de la loi d'exiger que les décideurs fassent abstraction de leurs connaissances acquises dans d'autres dossiers pour refuser la réclamation. Bien que le concept de stare decisis soit étranger au monde médical, vu l'évolution constante des connaissances scientifiques, il ne nous semble pas interdit de recourir au savoir acquis en étudiant des dossiers qui présentaient des faits similaires. Si, dans un dossier où des études scientifiques étoffées ont été produites, on a reconnu que le syndrome du tunnel carpien est une maladie caractéristique du travail de couturière, on devrait en venir aux mêmes conclusions dans le dossier d'une autre couturière qui est atteinte de ce syndrome et qui, faute de moyens, n'a pu produire ces études scientifiques. Le législateur souligne que le but de la Loi sur les accidents du travail et les maladies professionnelles est de réparer les lésions professionnelles ${ }^{40}$. Faut-il le rappeler, l'objectif initial lors de la création de la première Commission des accidents du travail du Québec en 1928 était d'assouplir les règles afin de faciliter les réclamations des travailleurs jusqu'alors frustrés par la rigidité du cadre des tribunaux de droit commun ${ }^{41}$.

Plusieurs questions nous viennent à l'esprit face à une telle situation:

40. L.R.Q. c. A-3.001, art. 1.

41. À ce sujet voir le rapport de la Commission Roy de 1923 Rapport de la Commission d'étude sur la réparation des accidents du travail, Québec, Imprimeur du Roi, 1925; voir aussi: K. Lippel, op. cit., note 24. 
Il est évident qu'un décideur peut consulter la jurisprudence non produite par les parties, mais peut-il tenir compte d'études épidémiologiques citées dans ces décisions pour admettre la réclamation du travailleur qui n'a pas produit ces études?

Lorsque l'assesseur médical a une idée préconçue sur une question médicale, n'est-il pas souhaitable qu'il soit obligé de l'exprimer dans le cadre de l'audition, afin que les parties puissent en prendre connaissance et y répondre s'il y a lieu?

Dans leur souci d'apparence d'impartialité, les décideurs se priverontils d'exercer leurs fonctions avec justice et équité?

D'un côté, il faut dénoncer tout mécanisme juridique qui obligerait les décideurs à s'aveugler volontairement sous prétexte que le système contradictoire exige qu'ils ne considèrent que les faits en preuve. De l'autre côté, il faut protéger l'ensemble des justiciables contre des initiatives personnelles qui permettraient aux décideurs de trancher en se basant sur des données que les parties et leurs experts n'ont pas eu l'occasion de commenter. Il est souhaitable que se développe un mécanisme qui permettrait aux décideurs de faire preuve d'une certaine souplesse sans que le droit d'être entendu soit brimé. Une modification aux règles de pratique des diverses instances obligeant les décideurs à soumettre aux parties des copies de toute étude ou tout document qu'ils estiment pertinents à la réclamation, assortie d'un délai raisonnable pour permettre aux parties de fournir leurs commentaires, assurerait une telle souplesse, tout en protégeant les justiciables contre les décisions basées sur des considérations étrangères à la preuve. Par contre, rendre formel un tel mécanisme pourrait inciter les tribunaux supérieurs à être plus sévères à l'égard de décideurs qui voudraient se baser sur des informations n'apparaissant pas dans la preuve.

Le processus d'indemnisation des victimes de lésions professionnelles et des victimes de la route n'est pas neutre. Qu'il s'agisse d'une décision de la Commission de la santé et de la sécurité du travail ou de la Société de l'assurance-automobile du Québec, les décideurs sont également les organismes payeurs, organismes qui ont des moyens supérieurs à ceux des victimes pour enquêter et étudier les questions médico-légales. Lorsqu'il s'agit d'une 
lésion professionnelle, le déséquilibre est encore aggravé par le fait que l'employeur a souvent un pouvoir économique qui lui permet de dépasser les capacités financières limitées du travailleur. Le droit n'est pas neutre non plus, pas même lorsqu'il est appliqué par des tribunaux administratifs agissant en appel, comme la Commission des affaires sociales et la Commission d'appel en matière de lésions professionnelles. Une application trop rigide des règles de droit conçues pour un contexte contradictoire basé sur l'égalité des parties peut à la limite produire une injustice structurelle qui risque de saboter la mission que le législateur se donnait en adoptant des législations d'indemnisation sans égard à la faute. Même si on doit rompre avec les traditions civilistes, il nous semble souhaitable d'exiger que les instances fassent bénéficier l'ensemble des justiciables de toute nouvelle donnée scientifique disponible, à la condition toutefois qu'il existe des mécanismes pour garantir que tous aient le droit de répondre à la documentation consultée par les décideurs.

c) Études inexistantes

Les conséquences de la «neutralité» officielle de nos tribunaux administratifs, souvent synonyme de passivité, s'apprécient davantage lorsqu'on fait la comparaison avec l'interventionnisme dont fait preuve le Tribunal d'appel des accidents du travail de l'Ontario. Une décision récente de ce tribunal illustre bien le contraste. Dans la Décision $859 / 89$, on évaluait l'opportunité de reconnaître la maladie pulmonaire obstructive chronique à titre de maladie professionnelle caractéristique d'un travail impliquant l'exposition à la poussière ${ }^{42}$. Dans un premier temps, le Tribunal chargé d'étudier la demande a suspendu l'enquête et a commandé une étude sur les pratiques de reconnaissance de cette maladie dans les autres provinces canadiennes, le tout aux frais du tribunal. Il a ensuite analysé la preuve qui comportait une certaine preuve épidémiologique. À la fin de cette démarche il conclut que la maladie pulmonaire obstructive chronique est une maladie professionnelle caractéristique d'un travail impliquant l'exposition à la poussière, et il commente la valeur d'une preuve épidémiologique en ces termes: 
«Les études épidémiologiques font l'analyse des liens statistiques existant entre les populations plutôt que l'étude des causes de cas particuliers de maladie. La preuve épidémiologique, lorsqu'elle existait, pouvait constituer une partie importante de la preuve servant à trancher les cas d'admissibilité; cependant, l'admissibilité ne pouvait dépendre exclusivement d'une preuve de cet ordre. Comme la production de preuves épidémiologiques prenait beaucoup de temps et était très dispendieuse, son absence ne devait pas être considérée comme indiquant l'absence de lien de causalité» ${ }^{43}$.

L'absence de preuve épidémiologique concluante n'implique pas le refus de la réclamation, du moins en Ontario. Au Québec, par contre, il y a lieu de se demander si la tendance de nos tribunaux n'est pas de rejeter la réclamation lorsqu'aucune étude épidémiologique n'est produite en preuve. On peut trouver, par exemple, des décisions qui refusent de reconnaître une relation entre le cancer et l'hydrazine au motif que les études démontrant une relation entre le produit et la maladie ont été effectuées sur des animaux ${ }^{44}$ ou encore qui rejettent la preuve épidémiologique parce qu'elle était effectuée sur des travailleurs exerçant un métier différent que celui du réclamant ${ }^{45}$. Les études épidémiologiques coûtent cher et rares sont les groupements de travailleurs ayant les moyens de les commanditer. De plus, lorsque le travailleur n'est pas syndiqué ou lorsqu'il travaille dans un domaine où on ne trouve que peu de salariés, il est illusoire de croire aux possibilités de produire des études épidémiologiques effectuées dans des conditions identiques. Par ailleurs, il existe des études qui sont commanditées par les fabricants de produits. Les sociétés ont plutôt intérêt, en règle générale, à ce que l'on démontre le caractère anodin des substances étudiées. Ainsi, on observe parfois que des chercheurs financés par l'industrie hésitent à rendre publics les résultats d'une recherche qui démontre qu'un produit est dangereux ${ }^{46}$.

43. Précitée, note 42 , p. 161 , cet extrait est tiré de la version française du résumé de la décision, car la décision elle-même a été rédigée en anglais. Il s'agit d'une traduction d'un extrait du jugement apparaissant à la page 175 .

44. Laforest c. Cie minière Québec-Cartier, [1989] C.A.L.P. 678.

45. Liboiron c. Gordon Sestock Construction Inc. et C.S.S.T., [1989] C.A.L.P. 925.

46. Sur l'ensemble de cette question voir: S. EPSTEIN, op. cit., note 2. 
En somme, il nous faut constater qu'en ce domaine, le travailleur est régulièrement confronté à une preuve trop coûteuse ou encore inaccessible. Une approche plus interventionniste de nos tribunaux permettait peut-être de réquilibrer la situation.

\section{Conclusion}

Nous avons touché à quelques aspects de l'interaction entre le droit et la science telle qu'elle se réalise à l'occasion du processus d'indemnisation. Un ensemble de facteurs rend extrêmement difficile la reconnaissance de certaines réclamations, particulièrement dans le domaine des maladies professionnelles. Ceux et celles qui rendent des décisions dans ce domaine doivent être conscients qu'exiger une preuve avec une certitude dite scientifique signifie, dans la majorité des cas, exiger l'impossible de ceux qui se disent malades à cause de leur travail. Il est impensable sinon impossible que des études s'effectuent sur chaque maladie dans chaque métier. L'exigence d'une telle preuve équivaudrait à un rejet de toute demande d'indemnisation présentée par des travailleurs et travailleuses oeuvrant dans de petites industries qui ne feront jamais l'objet d'étude. Le droit ne pose pas de telles exigences. Les politiques administratives ne le devraient pas non plus.

Faut-il le rappeler, les indemnités devraient être accordées lorsqu'il est probable que la maladie a été contractée au travail et qu'elle est reliée aux risques particuliers de celui-ci. À la limite on pourra se tourner vers la preuve épidémiologique pour démontrer que la maladie est caractéristique du travail mais cela ne serait nécessaire que dans la mesure où le travailleur n'aura pu établir l'existence d'un risque particulier. Si le travailleur établit l'existence d'un risque particulier, il ne devrait pas être nécessaire de démontrer qu'il y a d'autres travailleurs atteints de la même maladie et faisant le même travail.

Bref on peut dire que les spécificités de certaines approches médicolégales rendent difficile la reconnaissance du droit à l'indemnisation et ce, même dans les cas où la victime n'a qu'à prouver la relation entre sa lésion et son accident. Les particuliers qui réclament des prestations des régimes d'indemnisation sont désavantagés de par leurs moyens économiques, leur inexpérience dans le domaine et leurs capacités limitées d'affronter l'appareil 
bureaucratique de l'État. Une clarification de la confusion créée par l'interaction entre la médecine et le droit ne réglera pas l'ensemble des injustices causées par un déséquilibre structurel qui est peut-être inévitable. Il faut cependant espérer qu'un examen plus attentif et méticuleux du phénomène de la preuve médicale permettra aux décideurs de s'approcher davantage des objectifs initiaux du législateur: une juste réparation octroyée après un minimum de contentieux. 